\title{
Research On Wireless Electric Power Transfer By Fractional Frequency Excitation
}

\author{
Chunlai Yu, ${ }^{1, a, *}$, Xuecheng Zhu, Ziwei Gao ${ }^{1, \mathrm{c}}$, Chunbo Zhu ${ }^{2, \mathrm{~d}}$ \\ ${ }^{1}$ State Grid Heilongjiang Electric Power Company Limited Electric Power Research Institute, Xiangjiang \\ Street, Harbin, Heilongjiang Province, China \\ ${ }^{2}$ Electrical Engineering College, Harbin Institute of Technology, Xidazhi Street, Harbin, Heilongjiang \\ Province, China \\ an62fish@163.com, ${ }^{b}$ zhuxuecheng2004@163.com, ${ }^{\mathrm{c}}$ Gaoziwei1979@163.com, ${ }^{\mathrm{d}}$ zhuchunbo@hit.com \\ *corresponding author
}

Keywords: Wireless energy transfer, Driving source, Fractional frequency, Efficiency.

\begin{abstract}
An excitation mode for magnetic coupled resonant wireless electric power transfer was proposed, and its equivalent physical model was built. Mathematical expressions of transfer power and efficiency were built through analyzing operation mode and time-domain waveform thereof. Energy transfer characteristics and characteristics of different frequency dividing ratios were analyzed. Simulation and experiment showed that the transfer efficiency of the system can be improved by the mode, and the mode had certain application value.
\end{abstract}

\section{Introduction}

Resonance frequency improvement of resonance coils is beneficial to increase the transfer power in magnetic coupled wireless electric power transfer technology. However, the switching loss is increased rapidly with operating frequency increase of the driving source. The driving source has the largest loss in the system as a result ${ }^{[1-4]}$. Therefore, it is important to improve the resonance frequency of the resonance system and decrease the operating frequency of the driving source in the magnetic coupled wireless electric power transfer system. Time-domain analysis ${ }^{[5-8]}$ indicates that energy is transferred from one coil to another coil through the coupling effect of the resonance coil. The transfer is related to the resonance frequency and coupling coefficient closely. The driving source is used for supplementing energy to the coupling oscillation system on time within the transfer cycle only, but it is not involved in energy wireless transfer. It is not necessary to supplement energy to the resonance system in each cycle, and energy can be transferred in a wireless mode in the resonance system. In the paper, a fractional frequency energy transfer mode for high-frequency magnetic coupled resonant wireless electric power transfer is proposed on the basis of the thought.

\section{Analytical description of fractional frequency excitation energy transfer process}

The theoretical analysis model was shown in Fig.1. After one self-resonance cycle was completed, the energy of the resonance system was dissipated gradually under the action of resistance as a result of loss and load energy consumption. The resonance system was connected with an external excitation source, and energy was supplemented to the resonance system after an interval of several cycles. Energy was not supplemented to the resonance system in each cycle, which was supplemented regularly and intermittently after an fixed interval of several resonance cycles. The frequency of the driving source was $1 / n$ of the resonance frequency (n was a natural number greater than 1). Namely, the resonance frequency of the resonance system was just the fractional frequency of the driving source excitation frequency, wherein the resonance system was excited by the external driving source, and the contradiction between resonance circuit and driving circuit was alleviated. The coil resistance was less than the load resistance and internal resistance of the driving source usually, therefore it had relatively low influence on efficiency. 


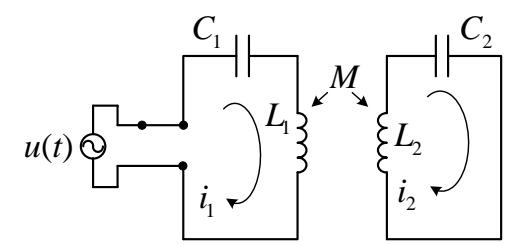

a) Excitation forced oscillation process

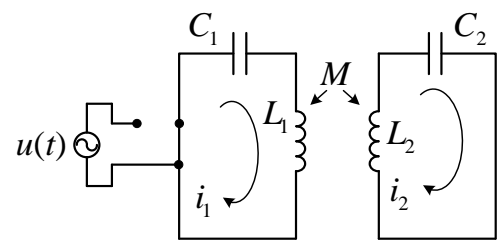

b) Excitation forced oscillation process

Figure 1 Theoretical analysis model.

Fractional frequency energy transfer was divided into two processes according to Fig. 1:

(1) Excitation forced oscillation process

External excitation was involved in system oscillation, and energy was supplemented to the coupling resonance system in the process. The system movement was the result of combined action in three aspects:

The first aspect was the influence of initial values in the system. Initial values included original inductance current and capacitor voltage. The time domain performance thereof was decay free oscillation caused by the initial values, and its amplitude was determined by the initial condition. The second aspect was the associated free oscillation of the resonance system caused by harmonic excitation, and the associated free oscillation was still available during zero initial state. The third aspect was steady-state response. The system responded by resultant oscillation of the three abovementioned movements when the driving source was just operated. The steady-state response was usually considered only during analysis on the forced oscillation response of the system. However, there was not enough time to decay the transient response since the research object was just in the intermittent operation.

$$
u(t)=U_{0} \cos \omega t
$$

The current response equation containing transient component in the transmitting coil should meet the follows ${ }^{[5]}$ :

$$
\begin{aligned}
i_{11}(t)= & e^{-\xi \omega_{0} t}\left(i_{01} \cos \omega_{d}+\frac{\dot{i}_{01}+\xi \omega_{0} i_{01}}{\omega_{d}} \sin \omega_{d} t\right)+ \\
& B \beta e^{-\xi \omega_{0} t}\left(\sin \theta \cos \omega_{d} t+\frac{\xi \omega_{0} \sin \theta-\omega \cos \theta}{\omega_{d}} \sin \omega_{d} t\right)+ \\
& B \beta \sin (\omega t-\theta)
\end{aligned}
$$

Wherein:

$\xi$-damping ratio;

$\omega$--resonance frequency;

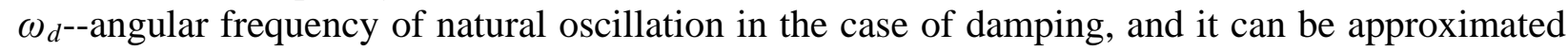
as follows at relatively small damping ratio:

$$
\omega_{d}=\omega_{0} \sqrt{1-\xi^{2}} \approx \omega_{0}
$$

$\omega$--harmonic excitation frequency;

$i_{01}, \dot{i}_{01}$--initial state parameter;

$B$--static displacement generated by the system under excitation $U_{0}$;

$\theta$--phase difference between response and excitation, $\theta=90^{\circ}$ when resonance frequency $\omega_{0}$ was equal to the frequency $\omega$ of harmonic excitation.

(2) Free-decay oscillation process

The system started free decay oscillation process after the excitation source was operated. The 
transmission coil was not connected with the driving source, and its quality factor was large. If only energy transfer was available and energy decay caused by coil self-loss was ignored, the transient process satisfied the follows ${ }^{[5]}$ :

$$
i_{12}(t)=A \cos \left(\frac{\omega_{0} \gamma}{2} t\right) \cos \left(\omega_{0} t\right)
$$

$\gamma$--coupling coefficient, $\gamma=M / \sqrt{L_{1} L_{2}}, \gamma \ll 1$.

Since free oscillation and incentive forced oscillation existed alternately, energy consumption in free decay oscillation was equal to energy increase in excitation forced oscillation when steady state was reached. The following relationship was met at the end of excitation forced oscillation (3):

$$
i_{12}\left(n \frac{2 \pi}{\omega_{0}}\right)=A \cos (n \gamma \pi)=i_{01}
$$

Wherein, $n$--ratio between total cycles and excitation cycles (frequency dividing ratio), $n=1$ indicated none self-oscillation cycle.

The load average power was shown as follows after one cycle:

$$
P_{\text {ave }}=\frac{\frac{L}{2}\left[i_{11}\left(\frac{2 \pi}{\omega_{0}}\right)\right]^{2}-\frac{L}{2} i_{01}^{2}}{n \frac{2 \pi}{\omega_{0}}}
$$

The average power of energy transfer under the fractional frequency excitation energy transfer mode was calculated according to formula (5) on the basis of current value.

\section{Experimental circuit}

The circuit of time-domain simulation analysis was based on a single-tube drive circuit structure ${ }^{[6]}$ as shown in Fig. 2

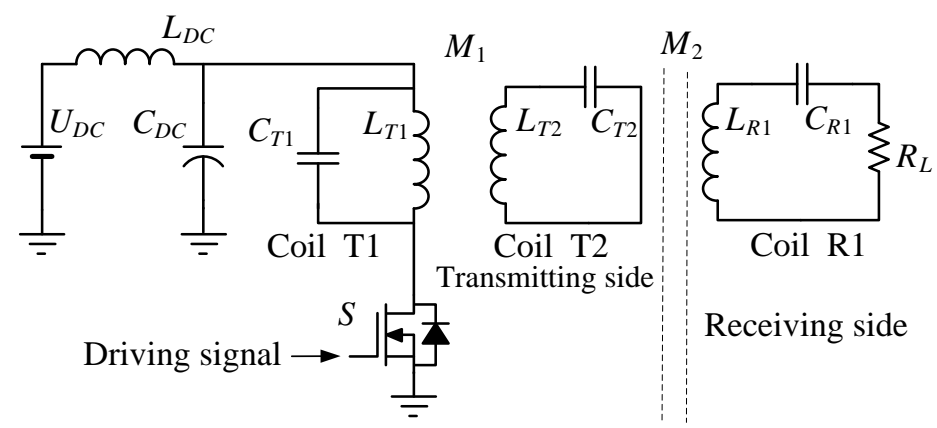

Figure 2 Circuit diagram.

Energy was supplemented to the resonance circuit when the switching tube was connected. The resonance circuit was oscillated freely when the switching tube was disconnected. Because resonance frequency and excitation frequency were matched well through design and regulation, specific fractional frequency trigger circuit was not required.

The simulation time domain results were shown in Fig. 2. Primary side and secondary side current had the following characteristics under different frequency dividing ratios $n$ : firstly, relatively large transient component was caused by power-on. The disturbance disappeared quickly after certain period of time. Secondly, the primary current amplitude was increased during operation of the driving source after waveform entered the working state of fractional frequency. The primary current amplitude was decreased gradually after excitation exit. The frequency dividing ratio was 
larger, the decrease was deeper. $23 \%$ of the initial amplitude was reached when $n=4$. Finally, the secondary current kept a stable amplitude basically during operation and suspension of the driving source. It was obvious that energy still can be transferred to the load through the wireless electric power transfer device when the excitation source was not operated.

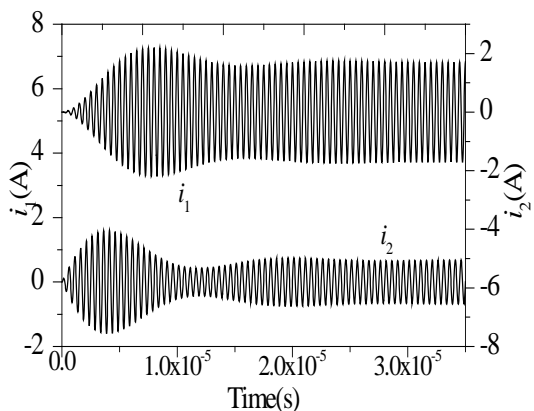

a) Waveform during $n=1$

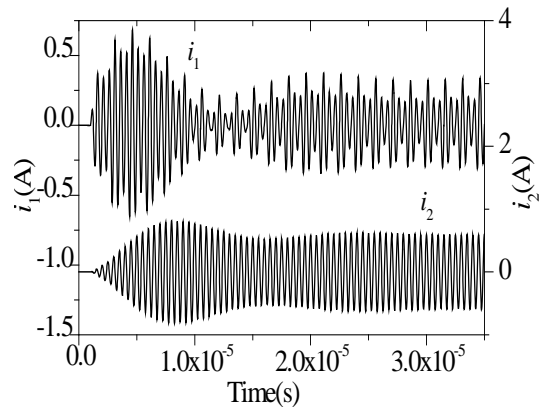

c) Waveform during $n=3$

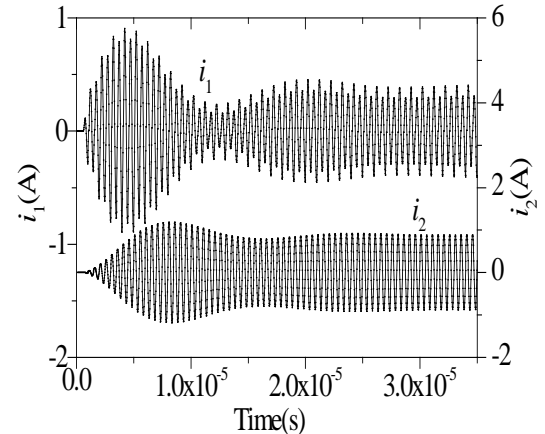

b) Waveform during $n=2$

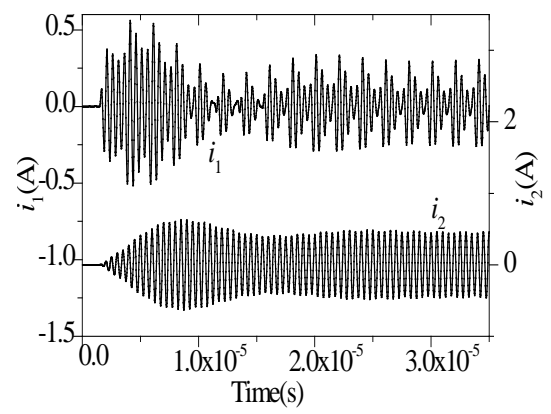

d) Waveform during $n=4$

Figure 3 Simulation waveform of fractional frequency energy transfer drive in time domain.

\section{Energy transfer characteristics under fractional frequency excitation}

The frequency dividing ratio=3 was selected respectively for obtaining the waveform, which was the same as simulation analysis in Fig. 4.
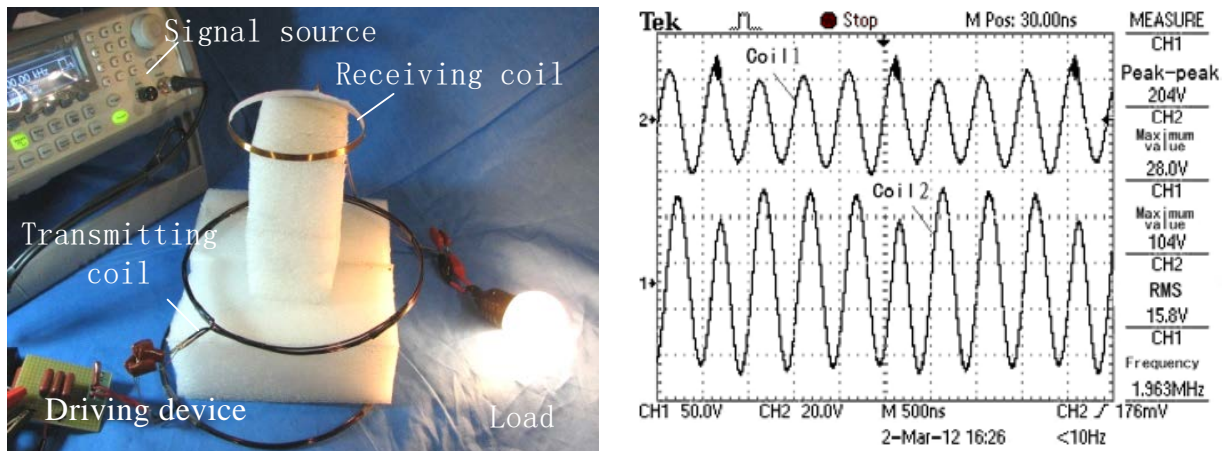

Figure 4 Measured device and waveforms of capacitor voltage.

Actual waveform was basically consistent with the simulation waveform. It was obvious that the working process of the actual circuit was consistent with the time-domain theoretical analysis, and corresponding power change at different driving frequencies was measured as shown in Fig. 5. 


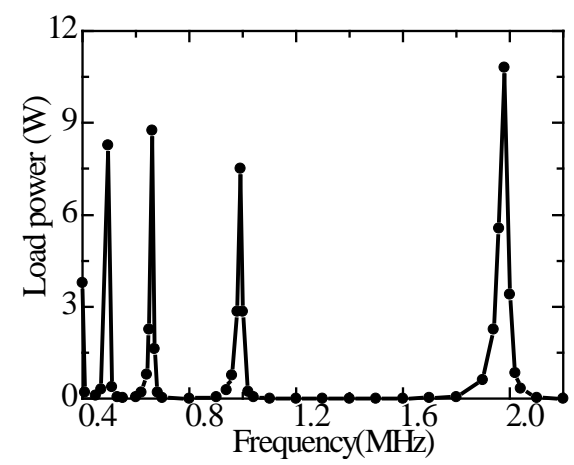

Figure 5 Curve of load power corresponding to frequency change.

Fig. 5 showed that the system was sensitive to the frequency deviation of the resonance circuit. The transfer power was the maximum when the driving source frequency was close to the resonance frequency of the coil 2. The output power was decreased rapidly after the resonance frequency was deviated. When the coil was operated near the resonance frequency $(1.98 \mathrm{MHz})$, the two divided-frequency $(992 \mathrm{kHz})$, the three divided-frequency $(660 \mathrm{KHZ})$ and the four dividedfrequency (496 KHZ), the transfer power reached relatively large value. The circuit was just in the fractional frequency energy transfer state.

\section{Waveform in the circuit under the working condition of fractional frequency excitation}

Different frequency dividing ratios $n$ were changed, the gate-to-source voltage $u_{d s}$ of the switching tube $S$ and the measured waveform of coil 2 capacitor voltage were obtained as shown in Fig. 6. The actual circuit waveform was consistent with theoretical analysis basically. $u_{d s}$ voltage frequency was equal to resonance frequency (as shown in Fig.6a) when the excitation frequency was equal to the resonance frequency. The voltage frequency $u_{d s}$ was smaller than the capacitor voltage frequency in coil 2 under the resonance state. The voltage spike with basically constant amplitude appeared, which also appeared at the free oscillation stage. Free oscillation was continuous before the power tube was opened next time, and the amplitude was decreased gradually.

$U_{\mathrm{ds}}$ did not have continuous free oscillation as shown in Fig. 6c) because of too long duty cycle.

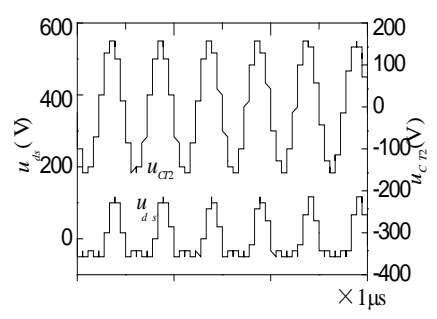

a) Voltage waveform during $n=1$

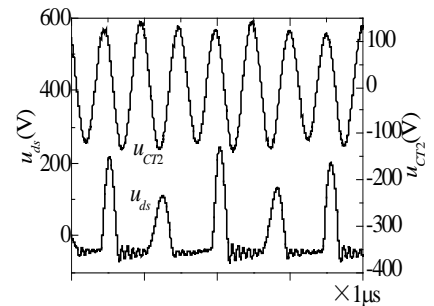

c) Voltage waveform during $n=3$

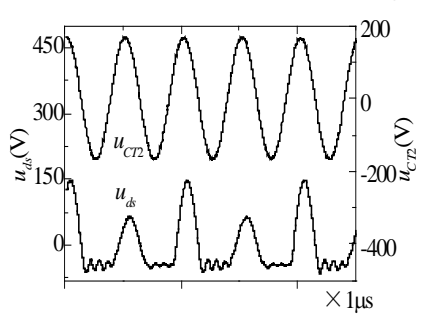

b) Voltage waveform during $n=2$;

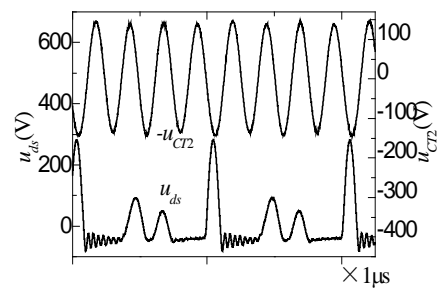

d) Voltage waveform during $n=4$

Figure 6 Voltage waveforms of $C_{\mathrm{T} 2}$ (upper) and $u_{\mathrm{ds}}$ under the condition of fractional frequency.

\section{Efficiency and power comparison at different frequency dividing ratios}

Experiment was carried out in the four above-mentioned frequency points. The connection time was kept constant (equal to the duration with duty ratio of $25 \%$ under the condition of none 
fractional frequency) at the same input voltage $(30 \mathrm{~V})$ and load resistance $(1 \mathrm{k} \Omega)$. Efficiency, output power and drain-source voltage peak value of the power tube were obtained as shown in Fig. 7.

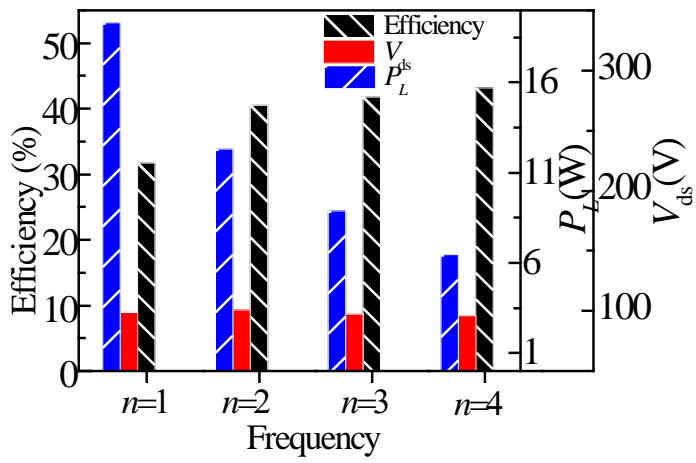

Figure 7 Output power, efficiency and drain-source voltage under the condition of fractional frequency.

Fig. 7 showed that the energy transferred to the resonance circuit was basically consistent each time since the opening duration of the driving source was consistent each time. The maximum voltage on the resonant capacitor was consistent basically, therefore the peak value of the drainsource voltage was basically the same. However, the load power was decreased significantly with the increase of the frequency dividing ratio because total energy was decayed gradually in the resonance process, and the power divided to the load was decreased. However, the overall efficiency was increased slightly with the decrease of the driving source working frequency, and the total efficiency was increased by $12 \%$ compared with that under the condition of fractional frequency.

The duty ratio of the power tube was fixed, while the working frequency of the driving source was reduced only to improve the transfer power. The opening time of the power tube was increased correspondingly, thereby compensating output power decrease caused by energy decrease at the self-resonance stage. The result was shown in Fig. 8. The gate-to-source voltage of the power tube was increased rapidly with frequency decrease. It was obvious that the output power was not increased linearly with the increase of the input power though the input power of the driving source was increased in each cycle. The reasons leading to the result were shown as follows according to analysis on the single-tube driving source during free oscillation: the initial energy was only related to the DC power voltage and resonant capacitor value during free oscillation though the opening time of the driving source was increased with $n$ increase. Therefore, the resonant energy was increased in the first cycle only through the method. The free oscillation transfer power was decreased with increase of the frequency dividing ratio. Therefore, there was a trend of output power decrease following frequency dividing ratio increase in Fig. 8.

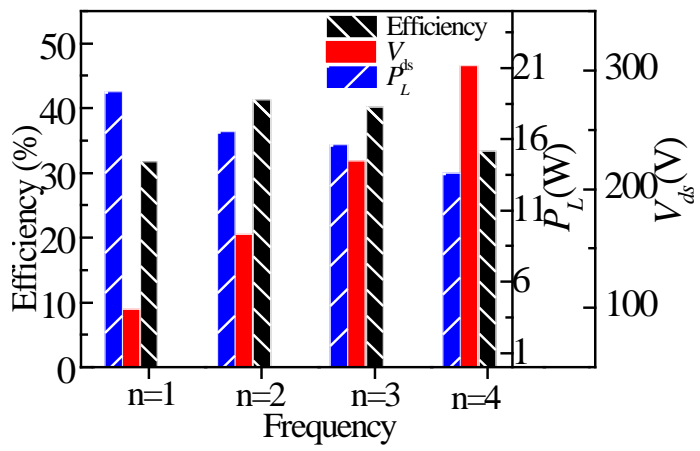

Figure 8 Output power, efficiency and drain-source voltage of power tube with gradual increase of input power to resonance system.

The maximum efficiency was discovered at two divided-frequency. The efficiency was decreased after increase of the frequency dividing ratio because the power injected into the 
resonance system was increased, but own loss was also increased after the increase of connection time. The total efficiency was decreased finally as a result.

\section{Conclusion}

The switching frequency of power components can be greatly decreased, or the resonance frequency of magnetic coupled coils can be greatly increased through the fractional frequency excitation energy transfer mode. The energy transfer mode is beneficial for reducing the loss of the driving source and improving the transfer efficiency of the system. The transfer distance of $15 \mathrm{~m}$, the maximum transfer power of $16.5 \mathrm{~W}$, and the efficiency of $41.3 \%$ can be realized at two dividedfrequency $(n=2)$. The efficiency is increased by $9.6 \%$ compared with that under the condition of none fractional frequency (the frequency is 31.7\% under the condition of none fractional frequency). The driving mode has certain prospect for the application situation with sensitive efficiency.

\section{References}

[1] CANNON B L, HOBURG J F, STANCIL D D, et al. Magnetic Resonant Coupling As a Potential Means for wireless electric power Transfer to Multiple Small Receivers [J]. IEEE Trans Power Electron, 2009, 24(7): 1819-25.

[2] Huang Xueliang, Tan Linlin, Chen Zhong, et al.Review and research progress on wireless electric power transfer technology [J], Transactions of China Electrotechnical Society, 2013, 28(10): $1-12$.

[3] Cheng Shijie, Chen Xiaoliang, Wang Junhua, et al. Key technology and applications of wireless electric power transfer[J]. Transactions of China Electrotechnical Society, 2015, 30(10): 67-84.

[4] Chu Jianglong, Li Yuling, Yang Shiyou. Design of power amplifier system for non-radiative magnetic resonance strongly coupled wireless electric power transfer[J]. Transactions of China Electrotechnical Society, 2013, 28(S2): 55-60.

[5] Zhang Xian, Yang Qingxin, Chen Haiyan, et al. Modeling and design and experimental verification of contactless power transfer system via electromagnetic resonnant coupling[J], Proceedings of the CSEE, 2012, 32(21): 153-158.

[6] Cheng Limin, Cui Yulong. Characteristics research on wireless electric power transfer systems using magnetic resonance coupling[J]. Transactions of China Electrotechnical Society, 2013, 28(S2): 23-27.

[7] KURS A, KARALIS A, MOFFATT R, et al. wireless electric power transfer via strongly coupled magnetic resonances [J]. Science, 2007, 317(5834): 83-6.

[8] TAK Y, PAR J, NAM S. Mode-Based Analysis of Resonant Characteristics for near-Field Coupled Small Antennas [J]. IEEE Antennas and Wireless Propagation Letters, 2009, 8(1238-41. 